\title{
Impact Analysis of GPS Tropospheric Error on Exterior Trajectory Measurement
}

\author{
DING Chuan-bing ${ }^{1, a, *}$, BIAN Wei-wei ${ }^{1, b}$, LI Hai-bin ${ }^{2, c}$ \\ ${ }^{1}$ China Ship Development and Design Center, Shanghai 201108, China \\ ${ }^{2}$ Beijing Machine and equipment Institute, Beijing, 100854, China \\ ash701fb@163.com, ${ }^{\mathrm{b}}$ 1hbmagician@hotmail.com, ${ }^{\mathrm{c}}$ karovie@163.com \\ *corresponding author
}

Keywords: Trajectory measure; Error source; GPS; Troposphere Delay; State parameters

\begin{abstract}
In order to improve the effect of trajectory control, it is necessary to enhance the accuracy of exterior trajectory measurement. Based on the particle dynamics model of the guided projectiles, various error sources in GPS positioning are discussed, and the mathematical model of tropospheric delay error is derived. The simulation of the GPS information received by the guided projectiles during flight is simulated by the Matlab. The impact of tropospheric delay error on the positioning error of GPS navigation trajectory is simulated. The research results provide some technical support for the error analysis of the GPS exterior trajectory measurement.
\end{abstract}

\section{Introduction}

As a weapon equipment identification system, high precision data processing is necessary. The data processing model should be established and calculated. After the data processing, the observation data can be filtered and a more accurate three dimensional position data can be obtained. Errors correction on the tropospheric refraction, ionospheric refraction, multipath effect, relativistic effect, clock error, SA frequency jitter, satellite orbit error, earth rotation correction, antenna phase center deviation, and earth fixed tide correction ${ }^{[1]}$ should be worked out before positioning solution. At present, the qualitative analysis of these above errors is more ${ }^{[2][3]}$, but the quantitative analysis is not much, which is unfavorable to the study of the error correction of multi trajectory measurement data. In order to improve the overall design of the shipborne weapon system and the precision of the trajectory estimation, the flight dynamics model of the missile particle trajectory and the position calculation model of the GPS missile borne receiver are established. Through the numerical calculation, the scheme trajectory of the projectiles is obtained. The Hopfield model and the Black model of troposphere delay error are established, and the simulation is carried out to analyze the relationship between the troposphere delay error and the flight exterior trajectory.

\section{The trajectory model for rocket projectile}

The projectile borne GPS receiver is used to track and detect the projectile flight, the data of the detected trajectory are processed, and the real time position and velocity of the projectile are calculated. The particle trajectory model can be solved by following equation, 


$$
\left\{\begin{array}{c}
\frac{d x}{d t}=v_{x} \\
\frac{d y}{d t}=v_{y} \\
\frac{d z}{d t}=v_{z} \\
\frac{d v_{x}}{d t}=-c H_{\tau}(y) G\left(v_{\tau}\right) v_{x} \\
\frac{d v_{y}}{d t}=-c H_{\tau}(y) G\left(v_{\tau}\right) v_{y}-g \\
\frac{d v_{z}}{d t}=-c H_{\tau}(y) G\left(v_{\tau}\right) v_{z}
\end{array}\right.
$$

The meanings of the symbols in equation (1) refer to the literature ${ }^{[4]}$.

\section{The model of position calculation for GPS projectile borne receiver}

The positioning solution equation of $T_{\mathrm{i}}$ is established in the earth coordinate system. Effect of atmospheric delay omitted, pseudo range observation equations can be written as follows ${ }^{[5,6]}$ :

$$
\rho_{i}^{j}(\mathrm{t})=R_{i}^{j}(t)+c \delta t_{i}(t)
$$

Where,

$$
\begin{aligned}
& R_{i}^{j}(t)=\left|\begin{array}{ll}
R_{i}^{j}(t)-R_{i}(t)
\end{array}\right|, \text { represent the geometric distances between stars; } \\
& R^{j}(t)=\left[\begin{array}{lll}
x^{j}(t) & y^{j}(t) & z^{j}(t)
\end{array}\right]^{T}, \text { represent the space rectangular coordinate vector of satellite } \mathrm{S}^{j}
\end{aligned}
$$
in the earth coordinate system;

$R_{i}(t)=\left[\begin{array}{lll}x_{i}(t) & y_{i}(t) & z_{i}(t)\end{array}\right]^{T}$, represent the space rectangular coordinates of observation station $T_{\mathrm{i}}$ in the earth coordinate system;

$\delta t_{i}(t)$, represent the GPS receiver clock error;

$C$, represent the light velocity.

The GPS receiver continuous observation of 4 satellites, and an equation from equation (2) can be written as follows:

$$
\left.\begin{array}{l}
\rho_{i}^{1}(t)=\left|R^{1}(t)-R_{i}\right|+c \delta t_{i}(t) \\
\rho_{i}^{2}(t)=\left|R^{2}(t)-R_{i}\right|+c \delta t_{i}(t) \\
\rho_{i}^{3}(t)=\left|R^{3}(t)-R_{i}\right|+c \delta t_{i}(t) \\
\rho_{i}^{4}(t)=\left|R^{4}(t)-R_{i}\right|+c \delta t_{i}(t)
\end{array}\right\}
$$

If the approximate position of the receiver is known, the offset between the real position $\left(x_{u}, y_{u}, z_{u}\right)$ and the approximate position $\left(\hat{x}_{u}, \hat{y}_{u}, \hat{z}_{u}\right)$ can be marked as $\left(\Delta x_{u}, \Delta y_{u}, \Delta z_{u}\right)$. 
The equation (4) can be expanded in the approximate position by the Taylor series, which can be written as follows:

$$
\left[\begin{array}{l}
\rho_{i}^{1}(t) \\
\rho_{i}^{2}(t) \\
\rho_{i}^{3}(t) \\
\rho_{i}^{4}(t)
\end{array}\right]=-\left[\begin{array}{llll}
l_{i}^{1}(t) & m_{i}^{1}(t) & n_{i}^{1}(t) & -1 \\
l_{i}^{2}(t) & m_{i}^{2}(t) & n_{i}^{2}(t) & -1 \\
l_{i}^{3}(t) & m_{i}^{3}(t) & n_{i}^{3}(t) & -1 \\
l_{i}^{4}(t) & m_{i}^{4}(t) & n_{i}^{4}(t) & -1
\end{array}\right]\left[\begin{array}{c}
\delta x_{i} \\
\delta y_{i} \\
\delta z_{i} \\
\delta \rho_{i}
\end{array}\right]+\left[\begin{array}{c}
R_{i 0}^{1}(t) \\
R_{i 0}^{2}(t) \\
R_{i 0}^{3}(t) \\
R_{i 0}^{4}(t)
\end{array}\right]
$$

Or

$$
a_{i}(t) \delta Z_{i}+l_{i}(t)=0
$$

The solution of the equation (6) can be written as follows:

$$
a_{i}(t) \delta Z_{i}+l_{i}(t)=0
$$

For the equation (2), the four unknown parameters can solved only by observing 4 satellites. In the course of observation, if the GPS receiver simultaneously tracks more than $n^{j}\left(n^{j}>4\right)$ satellites, the right side of the equation is no longer a 0 vector, but a residual vector $\boldsymbol{v}_{\mathrm{i}}(t)$, and the least squares method is used to solve the problem. Equation (6) can be written in the form of error equations as follows:

$$
\boldsymbol{a}_{i}(t) \delta \boldsymbol{Z}_{i}+\boldsymbol{l}_{i}(t)=\boldsymbol{v}_{i}(t)
$$

Where,

$$
\begin{aligned}
\underset{\left(n^{j} \times 1\right)}{\boldsymbol{v}_{i}(t)} & =\left[\begin{array}{llll}
v_{i}^{1}(t) & v_{i}^{1}(t) & \ldots & v_{i}^{n^{j}}(t)
\end{array}\right]^{T} \\
\underset{\left(n^{j} \times 4\right)}{\boldsymbol{a}_{i}(t)} & =\left[\begin{array}{cccc}
l_{i}^{1}(t) & m_{i}^{1}(t) & n_{i}^{1}(t) & -1 \\
l_{i}^{2}(t) & m_{i}^{2}(t) & n_{i}^{2}(t) & -1 \\
\vdots & \vdots & \vdots & \vdots \\
l_{i}^{n^{j}}(t) & m_{i}^{n^{j}}(t) & n_{i}^{n^{j}}(t) & -1
\end{array}\right]
\end{aligned}
$$

$n^{j}$, indicates the number of visible satellites;

$n_{t}$, represents the number of the epoch observations.

In the case of ignoring the variation of receiver clock error with time, Equation (7) can be written as follows:

$$
\boldsymbol{V}_{i}=\boldsymbol{A}_{i} \delta \boldsymbol{X}+\boldsymbol{L}_{i}
$$

Where,

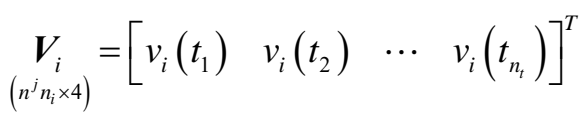

$$
\begin{aligned}
& \underset{\left(n^{j} n_{i} \times 4\right)}{\boldsymbol{A}_{i}}=\left[\begin{array}{c}
a_{i}\left(t_{1}\right) \\
a_{i}\left(t_{2}\right) \\
\vdots \\
a_{i}\left(t_{n_{t}}\right)
\end{array}\right] \\
& \underset{(4 \times 1)}{\delta \boldsymbol{X}_{i}}=\left[\begin{array}{llll}
\delta x_{i} & \delta y_{i} & \delta z_{i} & \delta \rho_{i}
\end{array}\right]^{T}
\end{aligned}
$$


According to the least squares adjustment solution, the position equation can be written as follows:

$$
\delta \boldsymbol{X}_{i}=-\left[A_{i}^{T} A_{i}\right]^{-1}\left[A_{i}^{T}(t) L_{i}\right]
$$

The real position equation can be written as follows:

$$
\boldsymbol{X}_{u}=\boldsymbol{X}^{\prime}+\delta \boldsymbol{X}_{i}=\boldsymbol{X}^{\prime}-\left[A_{i}^{T} A_{i}\right]^{-1}\left[A_{i}^{T} L_{i}\right]
$$

\section{Tropospheric delay error modeling}

The troposphere is the atmosphere below $50 \mathrm{~km}$, and $99 \%$ of the mass of the atmosphere is concentrated in this layer, which is also the main area of meteorological phenomena. The surface of the troposphere is exposed to the ground and the radiant heat energy is obtained from the ground. When the GPS signal passes through the troposphere, the propagation path is bent, that makes the measurement distance deviation. This phenomenon is called tropospheric refraction.

Tropospheric delay is the key factor affecting the accuracy of GPS positioning. Tropospheric delay refers to the refraction of electromagnetic waves by non electric atmosphere. In GPS positioning, the most commonly used models are Hopfield model and Black model ${ }^{[7,8]}$.

\subsection{Hopfield model}

Hopfield correction model is widely used in tropospheric refraction correction model:

$$
\delta \rho_{\text {trop }}=\frac{K_{d}}{\sin \left(E^{2}+6.25\right)^{1 / 2}}+\frac{K_{w}}{\sin \left(E^{2}+6.25\right)^{1 /}}
$$

Where

$$
\begin{gathered}
\left\{\begin{array}{c}
K_{d}=155.2 \times 10^{-7} \frac{P_{a}}{T_{a}}\left(h_{d}-h_{s}\right) \\
K_{w}=155.2 \times 10^{-7} \frac{4810}{T_{s}^{2}} e_{s}\left(h_{w}-h_{s}\right)
\end{array}\right. \\
\left\{\begin{array}{l}
h_{d}=40136+148.72\left(T_{s}-273.16\right) \\
h_{w}=11000
\end{array}\right.
\end{gathered}
$$

The water pressure at the observation station can be written as follows:

$$
e_{s}=e_{w}-4.5 \times 10^{-4}\left(1+1.68 \times 10^{-8} T_{w}\right)\left(T_{s}-T_{w}\right) .
$$

The saturated water vapor pressure can be written as follows:

$$
e_{w}=1013.246\left(\frac{373.16}{T_{w}}\right)^{5.02808} \cdot e^{-g\left(T_{w}\right)}
$$

Where,

$$
g\left(T_{w}\right)=g_{1}\left(T_{w}\right)+g_{2}\left(T_{w}\right)+g_{3}\left(T_{w}\right)
$$




$$
\left\{\begin{array}{l}
g_{1}\left(T_{w}\right)=18.19728\left(\frac{373.16}{T_{w}}-1\right) \\
g_{2}\left(T_{w}\right)=0.0187265\left\{1-\exp \left[-8.03945\left(\frac{373.16}{T_{w}}-1\right)\right]\right\} \\
g_{3}\left(T_{w}\right)=3.1813 \times 10^{-7}\left\{\exp \left[26.1205\left(1-\frac{373.16}{T_{w}}\right)\right]-1\right\}
\end{array}\right.
$$

$T_{s}, P_{s}, e_{s}$, and ${ }^{e_{w}}$ represent the temperature, pressure, water vapor pressure and saturated water pressure of the observation station respectively;

$h_{s}$ and $E$ represent the height and elevation of the observation station respectively;

air temperature adopts absolute temperature, and the unit is $K$;

the unit of the height is $\mathrm{m}$;

the unit of the elevation is $\left(^{\circ}\right)$.

\subsection{Black model}

Black model can be written as follows:

$$
\delta \rho_{\text {trop }}=K_{d}\left[\sqrt{1-\left(\frac{\cos E}{1+l_{0} \cdot h_{d} / R_{s}}\right)^{2}}-b(E)\right]+K_{w}\left[\sqrt{1-\left(\frac{\cos E}{1+l_{0} \cdot h_{w} / R_{s}}\right)^{2}}-b(E)\right.
$$

Where,

$E$, represents the satellite elevation;

$R_{s}$, represents the geocentric distance of observation station;

$T_{s}$, represents the temperature of observation station;

$K_{d}=0.002312\left(T-3.96_{s}\right) P_{a} / T_{s}$;

$K_{w}=0.0746512 e_{s} \cdot h_{w} / T_{s}^{2}$;

$e_{s}=E \cdot \exp \left(-37.2465+0.213166 T_{s}-0.256908 \times 10^{-3} T_{s}^{2}\right) h_{d}=148.98\left(T_{s}-3.96\right)$;

$h_{w}=11000$;

$T_{s}=t_{s}+273.16$;

$t_{s}$, the temperature of observation station; $\left({ }^{\circ} \mathrm{C}\right)$;

$l_{0}=0.167+\left[0.076+0.15 \times 10^{-3}\left(T_{s}-273.16\right)\right]^{-0.3 E} b(E)=1.92 /\left(E^{2}+0.6\right)$.

\section{4Simulation example and analysis}

In order to simulate the visible satellites, the rocket projectile flight trajectory three-dimensional scheme trajectory is solved according to a rocket projectile trajectory model, and then the number of visible satellites and each time point position are obtained, the satellite solution is worked out at last.

The meteorological conditions and satellite ephemeris briefly of the rocket projectiles launch site are known. The latitude is $45.004^{\circ}$, the longitude is $120.01^{\circ}$, and the height is $56.426 \mathrm{~m}$. 
The simulation results are shown in Figure 1 to figure 5.
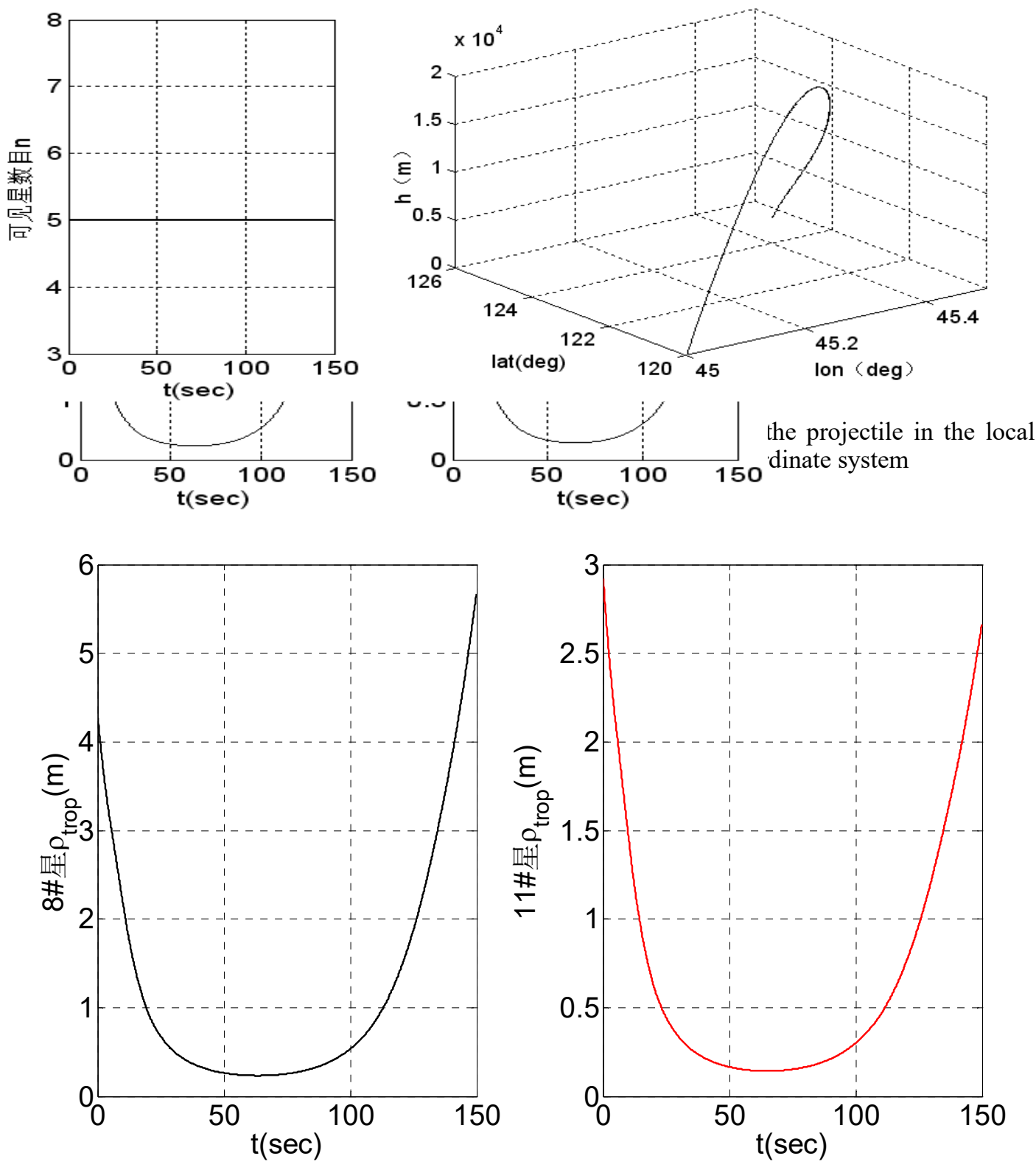

Fig. 3 the tropospheric delay error in $8 \#$ and $11 \#$ stars 

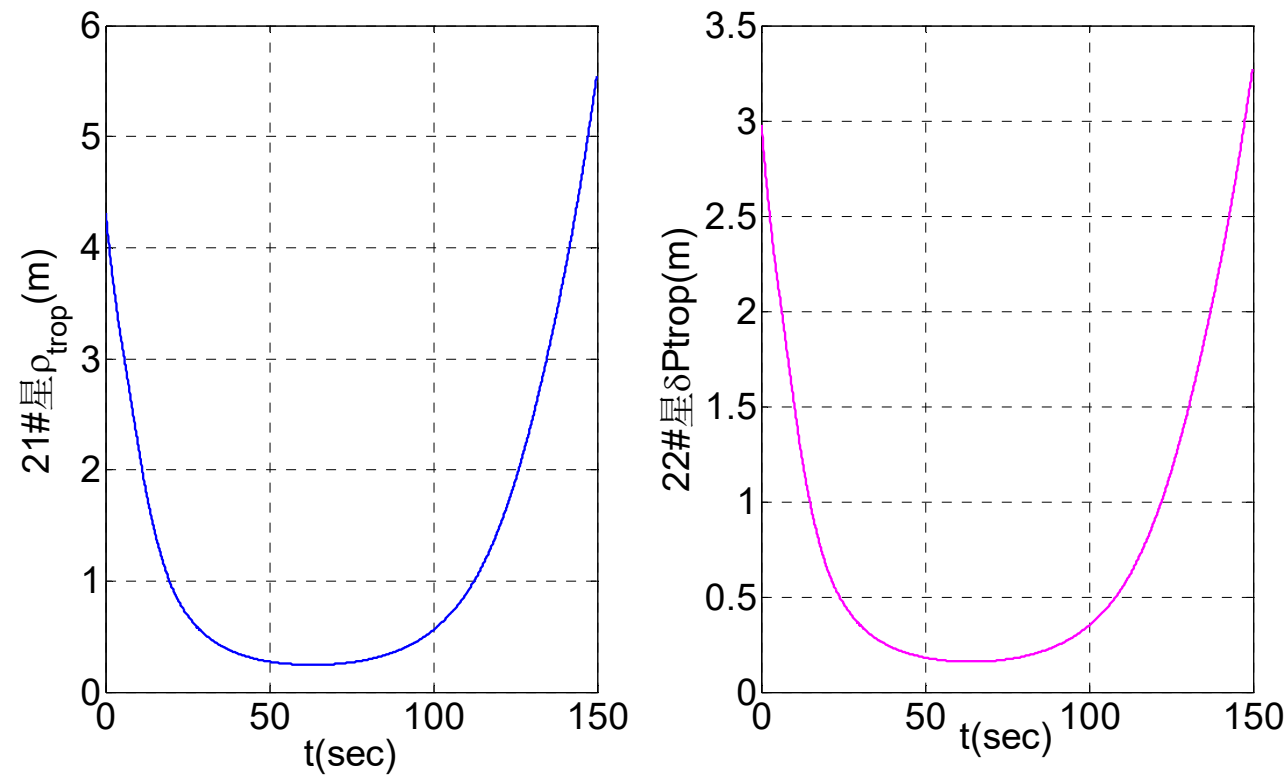

Fig. 4 the tropospheric delay error in $21 \#$ and $22 \#$ stars

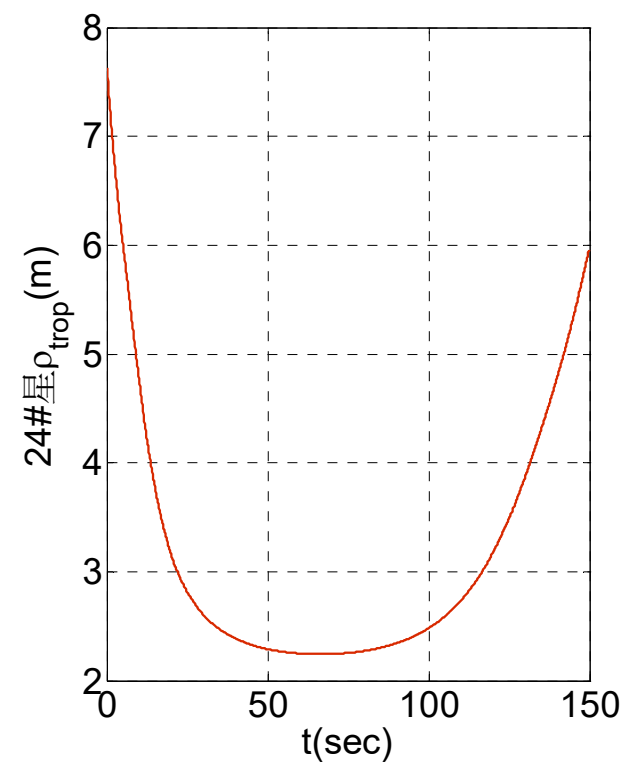

Fig. 5 the tropospheric delay error of $24 \#$ star

Since the simulation time is only 150 s, there is no change of stars, so the variation of pseudo range error caused by the tropospheric delay is relatively stable. If there is a change in the stars, the error will occur a large jump. In the 5 constellations, the 11\# star has the highest elevation angle, so the troposphere delay is the smallest; the 24\# star has the lowest elevation, so the tropospheric delay is the largest. According to the troposphere delay error model, the factors that affect the error are the elevation of the constellation and the flying height of the projectile. For a fixed constellation, the satellite elevation angle is not changed in a short time, so the height is the main factor that affects the error size, and the higher the value, the smaller the tropospheric delay error. As can be seen from the simulation Fig. 3 to Fig. 5, the initial segment and the end of the segment, the flying height of the projectile is small, so the error value is larger, and the middle segment, the flying height of the projectile is larger, and the error value is smaller. 


\section{Conclusion}

The particle trajectory model and the model of position calculation for GPS projectile borne receiver are established; the characteristics and causes of the troposphere are analyzed; the mathematical model of the tropospheric delay error for GPS positioning is established; the tropospheric delay error is simulated according to the scheme trajectory. The simulation results show that the factors that influence the tropospheric delay error are the elevation of the constellation and the flying height of the projectile. For a fixed constellation, the elevation angle of the satellite is not changed in a short time, so it is the main factor that affects the error. In the initial and the end of the projectile flight, the flying height of the projectile is small, so the error value is larger, and in the middle section, the flying height of the projectile is larger, and the error value is smaller. The results provide the basis of error analysis for the error correction of precise orbit determination and exterior trajectory data measurement.

\section{References}

[1] Xie Shijie, Liu Haojie. GPS Error Analysis[J]. Engineering of Surveying and Mapping, 1999, 8(3): 10-14. (in Chinese)

[2] Xu Yan, Yang Yuanxi, Xu Changguo. Analysis on Tropospheric Delay in Antarctic GPS Positioning[J]. Journal of Geodesy and Geodynamics, 2014, 34(1): 104-107. (in Chinese)

[3] Zhang Xiaofan, He Hong, Chen Jianfeng. GPStechnology in the Troposphere Delay Impact Analysis[J]. Geomatics \& Spatial Information Technology, 2014,37(2).187-192. (in Chinese)

[4] QIAN Xing-fang, LIN Rui-xiong, ZHAO Ya-nan. Missile Flight Dynamics[M]. Beijing: Beijing Institute of Technology Press, 2000. (in Chinese)

[5] Wang Huinan. Principles and Applications of GPS Navigation[M]. Beijing: Science Press, 2003. (in Chinese)

[6] Wei Ziqing, Ge Maorong. Mathematical Model of GPS Relative Positioning[M]. Changsha: Surveying and Mapping Press, 1998. (in Chinese)

[7] Wu Jiantong, Wang Licun, Yuan Jinsheng, etc. The Parameter estimation of GPS Error Model by Hopfield Neural Network Method[J]. Journal of Chinese Inertial Technology, 1998, 6(4): 24-75. (in Chinese)

[8] MOHAMED A H,SCHWARZ K P.A simple and economical algorithm for GPS ambiguity resolution on the fly using a white-filtering[J].Journal of the Institute of Navigation, 1998,45(3):221-231. 